\title{
Are cervical and breast cancer screening programmes equitable? The case of women with intellectual and developmental disabilities
}

V. Cobigo, ${ }^{1}$ H. Ouellette-Kuntz, ${ }^{2,3}$ R. Balogh, ${ }^{3,7}$ F. Leung, ${ }^{5}$ E. Lin ${ }^{3,6}$ \& Y. Lunsky ${ }^{3,4}$

1. School of Nursing Sciences, University of East Anglia, Norwich, UK

2. Community Health and Epidemiology, Queen’s University, Kingston, Ontario, Canada

3. Institute for Clinical Evaluative Sciences, Toronto, Ontario, Canada

4. Dual Diagnosis Program, Centre for Addiction and Mental Health, Toronto, Ontario, Canada

5. Dalla Lana School of Public Health, University of Toronto, Toronto, Ontario, Canada

6. Provincial System Support Program, Centre for Addiction and Mental Health, Toronto, Ontario, Canada

7. Faculty of Health Sciences, University of Ontario Institute of Technology, Oshawa, Ontario, Canada

This is the peer reviewed version of the following article: [Cobigo, V., Ouellette-Kuntz, H., Balogh, R., Leung, F., Lin, E., \& Lunsky, Y. (2013) Are cervical and breast cancer screening programmes equitable The case of women with intellectual and developmental disabilities. Journal of Intellectual Disability Research, 57(5), 478-488.] which has been published in final form at [10.1111/jir.12035]. This article may be used for non-commercial purposes in accordance with Wiley Terms and Conditions for SelfArchiving.

Correspondence: Dr Yona Lunsky, Centre for Addiction and Mental Health, Toronto, Ontario, Canada (email: yona.lunsky@ camh.ca). 


\begin{abstract}
Background Effective cancer screening must be available for all eligible individuals without discrimination. Lower rates of cervical and breast cancer screening have been reported in certain groups compared with women from the general population, such as women with intellectual and developmental disabilities (IDD). Research on the factors explaining those observed differences is crucial to deter- mine whether practices are unfair and could be improved. The aim of this population-based study was to describe cancer screening utilisation by women with IDD in Ontario, Canada compared with other women in Ontario. The specific objectives were (1) to estimate the rates of cervical and breast cancer screening among eligible women with IDD in Ontario; (2) to compare the rates of cervical and breast cancer screening between eligible women with and without IDD; and (3) to examine if any observed differences between women with and without IDD persist after factors such as age, socio- economic status, rurality and healthcare utilisation are accounted for. Method This study draws women with IDD from an entire population, and draws a randomly selected comparison group from the same population. It controls for important confounders in cancer screening within the limitations of the data sources. The study was conducted using health administrative databases and registries in Ontario, Canada. Two cohorts were created: a cohort of all women identified as having an IDD and a cohort consisting of a random sample of $20 \%$ of the women without IDD. Results The proportion of women with IDD who are not screened for cervical cancer is nearly twice what it is in the women without IDD, and 1.5 times what it is for mammography. Conclusions Findings suggest that women with IDD experience inequities in their access to cancer screening. Public health interventions targeting this population should be implemented.
\end{abstract} Keywords: administrative data, healthcare disparities, intellectual disability, population-based study 


\section{Introduction}

Effective screening tests are available for both cervical and breast cancers, which allow for the early detection of precancerous lesions and cancers and lead to reductions in cancer incidence and mortality (Boyle \& Levin 2008). Such resources should be available to all eligible women without discrimination. Despite efforts to remove barriers to cancer screening and healthcare access, disparities remain, especially among traditionally underserved populations (Eaker et al. 2001). Recent immigrants (particularly those from visible minorities), persons with low income and education, as well as limited literacy skills tend to have lower rates of screening (Lofters et al. 2007; Spadea et al. 2010). Living in a rural area is another variable potentially explaining lower rates of screening because of longer distances to access healthcare facilities (Eaker et al. 2001).

Individuals' variations in healthcare resources utilisation is also likely to impact cancer screening uptake (Lofters et al. 2007, 2010). General practitioners (GP) not recommending cancer screening to their patients also decreases the likelihood of an individual being screened (Finney Rutten et al., 2004). Finally, patients' lack of awareness of screening programmes and their benefits, beliefs and fears towards the procedure, as well as communication problems with healthcare professionals have been associated with lower rates of screening (Finney Rutten et al. 2004; Spadea et al. 2010).

In this regard, persons with intellectual and developmental disabilities (IDD) are particularly vulnerable to inequitable access to cancer screening; they tend to be poorer, have limited literacy and communication skills, and often do not understand the procedure and its benefits (Willis et al. 2008; Wilkinson et al. 2011). In addition, health professionals tend to report insufficient training and negative attitudes towards patients with IDD (Broughton 2002; Willis et al. 2008). Lower rates of cervical and breast cancer screening have been consistently reported in women with IDD compared with women without IDD. Studies in the UK and the USA reported that the proportion of women with IDD not receiving Papanicolau (Pap) smears is 3-5 times that found in the general population (Havercamp et al. 2004; Reynolds et al. 2008). Similar findings are reported for breast cancer screening. In the USA, the proportion of women with IDD not screened for breast cancer was found to be 2.1 times what it is in women without IDD (Havercamp et al. 2004), while in Australia, one study suggested the proportion of women with IDD not receiving mammography was 1.4 times the proportion of women in the general population (Sullivan et al. 2003).

The existing literature, while informative, is still limited (Willis et al. 2008) and must be replicated to compare findings in countries with different service delivery models and policies that might impact the uptake of cancer screening in women with IDD. First, IDD samples are often not representative of the entire IDD population because they are drawn from users of services for persons with IDD (Willis et al. 2008). Consequently, they do not account for individuals not receiving IDD services (e.g. Stein \& Allen 1999; Davies \& Duff 2001; Sullivan et al. 2003; Havercamp et al. 2004; Biswas et al. 2005; Gesualdi 2006; Lalor \& Redmond 2009). This is an important limitation, as we can speculate that persons receiving IDD services are likely to be more frequently oriented towards appropriate health care than those unknown to IDD services. In addition, women living with family caregivers tend to have the most limited knowledge about cancer screening, compared with those living in paid residential set- tings or living alone or with a spouse (Parish et al. 2012b). 
A population-based approach is thus recommended to draw findings representative of the whole population with IDD (Parish \& Saville 2006). However, identification of individuals with IDD may be more difficult when not relying on registries of IDD service users. For example, Parish \& Saville (2006) used a population-based approach to study health disparities. Using a national survey, they identified 296 women with cognitive limitations defined as having experienced (1) confusion or memory loss; (2) problems making decisions; or (3) who required supervision for their own safety. Authors noted that findings must be considered with great caution as they might not be specific to persons with IDD.

When describing cancer screening uptake in women with IDD, it is crucial to compare with data from the general population in order to appraise the extent of the disparities. Previous studies either compared with publicly available screening rates in the general population (e.g. Stein \& Allen 1999; Davies \& Duff 2001; Sullivan et al. 2003; Biswas et al. 2005; Lalor \& Redmond 2009), or they identified a comparison group using a different method than the one used to select the IDD group (e.g. Havercamp et al. 2004). Thus, observed differences between the IDD and the non-IDD groups may be explained by differences in the methods used rather than true differences in access. Finally, published studies in IDD rarely controlled for important con- founders in cancer screening such as age, socio- economic status, rurality or healthcare utilisation (Eaker et al. 2001; Lofters et al. 2007, 2010; Spadea et al. 2010). For example, Reynolds et al. (2008) identified women aged 25-64 in three Primary Care Trusts, from which they selected the group with IDD based on clinical records of Learning Disabilities Teams and GP. Controls were women from the same GP practice and within \pm 5 years of age, but investigators did not control for any other variables.

There are consequently questions about whether the published findings apply only to a subgroup of women with IDD and whether they might be because of factors other than IDD. For these reasons, we designed a study that (1) draws women with IDD from an entire population; (2) draws a randomly selected comparison group from the same population; and (3) controls for important con- founders within the limits of the data sources.

The aim of this population-based study was to describe cancer screening utilisation by women with IDD in Ontario, Canada compared with other women in Ontario. The specific objectives were (1) to estimate the rates of cervical and breast cancer screening among eligible women with IDD in Ontario; (2) to compare the rates of cervical and breast cancer screening between eligible women with and without IDD; and (3) to examine if any observed differences between women with and without IDD persist after factors such as age, socio- economic status, rurality and expected use of healthcare resources are accounted for.

\section{Methods}

\section{Study setting}

In Canada, health care is a provincial jurisdiction and each province provides universal health coverage to its residents. Ontario, Canada's most populated province (13 million habitants, one-third of the Canadian population) has screening programmes for cervical and breast cancers, in accordance with national and international recommendations. The Ontario Cervical Screening Program, launched in 2000, has guidelines stating that women aged 20-69, who have been sexually active, should have a Pap test every 3 years (Cancer Care Ontario 2000). Pap tests are conducted by family physicians or other healthcare providers during women's regular physical examinations. The Ontario Breast Screening Program 
(OBSP), which was established in 1990, provides biannual mammograms for women aged 50 and older, but specifically targets women between the ages of 50 and 69 (Cancer Care Ontario 2010). As an organised screening programme, the OBSP sends recall notices, communicates screening results to the women, and helps initiate specialist care for women with abnormal mammograms. Canadian Consensus Guidelines on the primary care of adults with IDD (Sullivan et al. 2011) also recommend regular screening tests for cervical and breast cancers in women with IDD, in accordance with the Cancer Care Ontario guidelines.

\section{Data sources and management}

Data for this study were accessed from the Institute for Clinical Evaluative Sciences (ICES). ICES houses several anonymised, linked health-related databases on Ontarians eligible for health services through a research agreement with the Ministry of Health and Long-Term Care. We obtained approval to conduct this study from the Research Ethics Boards at Sunnybrook Health Science Centre and the Centre for Addiction and Mental Health, Toronto, Ontario.

There were nine sources of information used in this study, including five health administrative data- sets, three registries, and census data. The administrative datasets cover inpatient hospital discharges (Canadian Institute for Health Information- Discharge Abstract Database), same-day surgeries (Same-day Surgery Database), emergency department visits (National Ambulatory Care Reporting System), inpatient mental health bed discharges (Ontario Mental Health Reporting System), and fee-for-service physician claims (Ontario Health Insurance Plan). These five datasets capture the vast majority of the formal medical services, which all legal residents of Ontario are eligible to receive. The datasets are used by the single insurance payer (the Ontario Government) for funding and reimbursement purposes. They include data on demographics, clinical information, and diagnoses.

Demographic variables used in our analyses (age, sex, and postal code) were provided by the three registries which cover all Ontario residents eligible for the province's universal healthcare insurance plan (Registered Persons Database), as well as demographic and clinical information on all residents who are diagnosed with cancer or have died of cancer (Ontario Cancer Registry) and on women eligible for the OBSP, including whether and when they received a mammogram. The 2006 Canadian Census was used to provide information on household income and rurality based on the individual's postal code (i.e. first three digits). Race and ethnicity, while potentially important contributors to screening, could not be studied as the information was not available in these databases. Scrambled healthcare numbers were used as unique identifiers to link the data- bases in a manner to preserve anonymity. Linking with the census was possible using the first three digits of postal codes.

Two cohorts of adult women living in Ontario who were continuously eligible for health coverage over a 1-year period (1 April 2009 to 31 March 2010) were created. An IDD cohort was identified based on the diagnostic information provided in the five health administrative datasets ( $\mathrm{n}=17 \mathrm{777})$. The method for identifying individuals with IDD is detailed in (Lin et al. 2012). In brief, all International Classification of Disease (ICD)-9, ICD-10 and Diagnostic and Statistical Manual of Mental Disorder (DSM) codes or their database equivalents were included that reflected conditions consistent with our provincial government's eligibility criteria for disability support because of an IDD (Service Ontario 2008). The list of specific IDD diagnostic codes is available in (Lin et al. 2012) or directly from the authors. Women were counted 
as having an IDD when one of the listed diagnostic codes appeared at least twice in the physicians' billing claims, or once in any other datasets, since the date of inception of each dataset. A comparison cohort of Ontarian women was created from a random sample of $20 \%$ of the general population without IDD ( $\mathrm{n}=1$ 440 962). A sample of $20 \%$ was used as it provides the power necessary for any analysis, while reducing the computing requirements (Table 1). To examine cervical cancer screening, women in the IDD and nonIDD cohorts who were 20-69 years of age on 1 April 2009 were included. Women from the Ontario Cancer Registry with a gynaecological cancer diagnosis prior to 1 January 2010 were excluded as they were more likely to receive a Pap smear for diagnostic purposes.

Women who had undergone a hysterectomy prior to 31 March 2010 were also excluded. An algorithm consisting of billing codes submitted either by a physician performing a Pap test (G365, G394, E430) or a cytopathologist interpreting the Pap test (L812, L713) was used to determine if a woman had been screened. The proportions of women in each cohort who had at least one Pap test over a 3-year period (1 April 2007-31 March 2010) were then calculated.

For the analysis of breast cancer screening, women in the IDD and non-IDD cohorts who were 50-69 years of age on 1 April 2009 were included. Women with a diagnosis of breast cancer from the Ontario Cancer Registry were excluded as they were more likely to have a mammogram for reasons other than screening. Women who had undergone a mammogram were identified from records in the OBSP and from billing codes submitted by physicians (X185). The proportions of women in each cohort who received at least one mammogram over a 2-year period (1 April 2008-31 March 2010) were then calculated.

\section{Statistical analysis}

Frequencies and proportions of women with and without IDD who had received cervical and breast cancer screening (respectively) were calculated across age, rurality, income, and expected use of healthcare resources. Age was categorised into 10-year intervals using 1 April 2009 as the reference date. Using population numbers from Statistics Canada, persons living in a community with a population less than or equal to 10000 were designated rural; otherwise they were considered urban (Statistics Canada 2007). Household income deter- mined using individuals’ postal codes were used to rank the 
Table 1. Demographics of women with and without intellectual or developmental disability (as of 1 April 2009 - 31 March 2010), by age, rurality, income, resources utilization band, Ontario

\begin{tabular}{|c|c|c|c|c|c|}
\hline & \multicolumn{2}{|c|}{$\begin{array}{c}\text { Women with intellectual or developmental } \\
\text { disability (total } n=17777 \text { ) }\end{array}$} & \multicolumn{2}{|c|}{$\begin{array}{c}\text { Women without intellectual or developmental } \\
\text { disability (total } n=1440962 \text { ) }\end{array}$} & $\begin{array}{c}\text { P- } \\
\text { Value }\end{array}$ \\
\hline All & 17777 & 100.0 & 1440962 & 100.0 & $<0.001$ \\
\hline $20-29$ years & 4427 & 24.9 & 284211 & 19.7 & \\
\hline 30-39 years & 3682 & 20.7 & 304486 & 21.1 & \\
\hline 40-49 years & 4345 & 24.4 & 347828 & 24.1 & \\
\hline 50-59 years & 3495 & 19.7 & 298217 & 20.7 & \\
\hline Rural & 2752 & 15.5 & 158248 & 11.0 & \\
\hline Urban & 15025 & 84.5 & 1282714 & 89.0 & \\
\hline Income Quintile (\%) & & & & & $<0.001$ \\
\hline Missing & 169 & 1.0 & 24792 & 1.7 & \\
\hline Quintile 1 - Low & 4934 & 27.8 & 270458 & 18.8 & \\
\hline Quintile 2 & 3739 & 21.0 & 279362 & 19.4 & \\
\hline $0=$ non-users & 800 & 4.5 & 99455 & 6.9 & \\
\hline $1=$ healthy users & 400 & 2.3 & 65258 & 4.5 & \\
\hline $2=$ low morbidity & 1658 & 9.3 & 212108 & 14.7 & \\
\hline $3=$ moderate morbidity & 9118 & 51.3 & 753723 & 52.3 & \\
\hline $4=$ high morbidity & 3947 & 22.2 & 266522 & 18.5 & \\
\hline $5=$ very high morbidity & 1854 & 10.4 & 43896 & 3.1 & \\
\hline
\end{tabular}


poorest to the wealthiest census area of Ontario and group them into quintiles (poorest $=1$; wealthiest $=5$ ) (Statistics Canada 2009). The role of healthcare professionals in recommending screening is central in educating women and increasing its uptake, especially among traditionally underserved groups (Finney Rutten et al. 2004; Spadea et al. 2010). As a consequence, expected use of healthcare resources appeared to be an important variable to include in the analyses. The Resource Utilization Bands (RUB) is a value that ranges from 0 to 5 to categorise individuals based on their expected use of healthcare resources, with 0 being the lowest expected healthcare utilisation and 5 the highest expected healthcare utilisation (The Johns Hopkins University Bloomberg School of Public Health 2005; CHSPR 2008). Following a standard and validated method, the RUB is determined based on a combination of information on age, sex and diagnoses (as reported in the administrative datasets in the 2 years preceding the dependent variable's observation window).

The association between IDD status and screening (i.e. Pap or Mammography) was assessed using logistic regression to calculate the odds ratios for both the unadjusted association and the association after control variables (age, rurality, income quintile, and RUB) were introduced into a multivariate model.

\section{Results}

Table 2 compares the proportions of women with and without IDD who had a Pap test over 3 years, as well as the proportions by age, rurality, income and RUB. About 34\% of women with IDD aged 20-69 received a Pap test over 3 years (out of 16663 eligible women), compared with 66.8\% of women without IDD. Thus, the proportion of age- eligible women with IDD in Ontario who are not screened for cervical cancer is nearly twice what it is in the women without IDD. The logistic regression examining the unadjusted relationship between IDD status and Pap test provided a crude odds ratio showing the association between IDD and Pap test to be 0.26 (95\% CI 0.25, 0.27). This difference remained after controlling for age, rurality, income quintile and RUB (OR = 0.21, 95\% CI 0.20, 0.21).

Similar, albeit less dramatic, differences were found for breast cancer screening (Table 3). About $42 \%$ of the women with IDD aged 50-69 received a mammogram over a 2-year period (out of 5171 eligible women), compared with $60 \%$ of women without IDD. The proportion of age-eligible women with IDD in Ontario who are not screened is thus 1.5 times the proportion for women without IDD. Proportions of women who had a mammogram remains inferior in the IDD cohort compared with women from the general population, even when comparing them by age groups, rurality status, income quintile of the neighbourhood where they live, and expected use of healthcare resources.

Adjusting for control variables had very little effect on the odds ratio for breast cancer screening. The crude odds ratio showing the association between IDD and mammogram uptake was 0.47 (95\% CI 0.45, $0.50)$, while the adjusted odds ratio was 0.46 (95\% CI 0.43, 0.49).

\section{Discussion}

This study compared population rates of cervical and breast cancer screening in women with IDD and a

large random sample of women without IDD. Women with IDD were found to be nearly twice as likely to 
Table 2. Proportion of women with and without intellectual or developmental disability who had at least one Pap test screening over three years (1 April 2007 - 31 March 2010), by age, rurality, income, resources utilization band, Ontario

\begin{tabular}{|c|c|c|c|c|}
\hline & \multicolumn{2}{|c|}{$\begin{array}{c}\text { Women with intellectual or developmental } \\
\text { disability (total } n=16663 \text { ) }\end{array}$} & \multicolumn{2}{|c|}{$\begin{array}{c}\text { Women without intellectual or developmental } \\
\text { disability }(n=1352366)\end{array}$} \\
\hline \multicolumn{5}{|l|}{ All } \\
\hline & 16663 & 34.3 & 1352366 & 66.8 \\
\hline Age (\%) & 4389 & 33.0 & 283857 & 65.7 \\
\hline 20-29 years & 3535 & 38.5 & 299530 & 72.1 \\
\hline 30-39 years & 3970 & 39.4 & 321781 & 71.4 \\
\hline 40-49 years & 3117 & 30.1 & 260862 & 65.9 \\
\hline Rurality (\%) & 14109 & 35.1 & 1209291 & 66.8 \\
\hline \multicolumn{5}{|l|}{ Rural } \\
\hline & 158 & 20.9 & 23903 & 26.5 \\
\hline Urban & 4599 & 38.3 & 254197 & 60.7 \\
\hline Income Quintile (\%) & 3485 & 33.3 & 261508 & 64.9 \\
\hline & 781 & 4.4 & 96910 & 15.9 \\
\hline Quintile 4 & 389 & 24.4 & 63536 & 57.8 \\
\hline Quintile 5 - High & 1597 & 25.4 & 204279 & 62.1 \\
\hline Resource utilisation band (\%) & 8607 & 32.7 & 700964 & 71.9 \\
\hline $0=$ non-users & 3649 & 48.7 & 249499 & 79.2 \\
\hline 1 = healthy users & 1640 & 35.5 & 37178 & 59.5 \\
\hline \multicolumn{5}{|l|}{2 = low morbidity } \\
\hline & 16663 & 34.3 & 1352366 & 66.8 \\
\hline $3=$ moderate morbidity & 4389 & 33.0 & 283857 & 65.7 \\
\hline 4 = high morbidity & 3535 & 38.5 & 299530 & 72.1 \\
\hline 5 = very high morbidity & 3970 & 39.4 & 321781 & 71.4 \\
\hline
\end{tabular}


Table 3. Proportion of women with and without intellectual or developmental disability who had at least one mammogram over 2 years (1 April 2008 - 31 March 2010), by age, rurality, income, resources utilization band, Ontario

\begin{tabular}{|c|c|c|c|c|}
\hline & \multicolumn{2}{|c|}{$\begin{array}{l}\text { Women with intellectual or developmental } \\
\text { disability (total } n=5 \text { 171) }\end{array}$} & \multicolumn{2}{|c|}{$\begin{array}{c}\text { Women without intellectual or developmental } \\
\text { disability (488 545) }\end{array}$} \\
\hline All & 5171 & 41.6 & 488545 & 59.9 \\
\hline 20-29 years & 1748 & 41.6 & 197213 & 62.2 \\
\hline \multicolumn{5}{|l|}{ 30-39 years } \\
\hline & 957 & 39.6 & 65193 & 60.5 \\
\hline 60-69 years & 1499 & 41.4 & 85372 & 53.5 \\
\hline Rurality (\%) & 1024 & 41.1 & 94256 & 58.0 \\
\hline Rural & 918 & 41.2 & 95094 & 60.3 \\
\hline Urban & 897 & 41.8 & 100989 & 62.7 \\
\hline Income Quintile (\%) & 788 & 43.4 & 107676 & 65.2 \\
\hline \multicolumn{5}{|l|}{ Missing } \\
\hline & 184 & 8.7 & 25699 & 14.5 \\
\hline Quintile 5-High & 826 & 39.2 & 25711 & 60.0 \\
\hline Resource utilisation band (\%) & 5171 & 41.6 & 488545 & 59.9 \\
\hline $0=$ non-users & 3423 & 41.5 & 291332 & 58.2 \\
\hline $1=$ healthy users & 1748 & 41.6 & 197213 & 62.2 \\
\hline $2=$ low morbidity & 957 & 39.6 & 65193 & 60.5 \\
\hline $3=$ moderate morbidity & 4214 & 42.0 & 423352 & 59.8 \\
\hline $4=$ high morbidity & 45 & 28.9 & 5158 & 21.9 \\
\hline $5=$ very high morbidity & 1499 & 41.4 & 85372 & 53.5 \\
\hline
\end{tabular}


not be screened for cervical cancer. While alarming, it is below than rates observed in the UK and the USA where the proportion of women with IDD not receiving a Pap test has been reported to be 3-5 times (respectively) that found in the general population (Havercamp et al. 2004; Reynolds et al. 2008). In Ontario, we found that the proportion of women with IDD not screened for breast cancer is 1.5 times the proportion of women without IDD, which is similar to the rates reported in an Australian study (Sullivan et al. 2003). However, in the USA, bigger differences have been observed, with women with IDD twice as likely to not have a mammography than women without IDD (Havercamp et al. 2004). Different models of healthcare delivery and policies, such as a universal healthcare coverage or organised screening programmes, might explain variations between countries. Some variation may also be attributed to differences in how data were obtained between studies, for both the group with IDD and the control group. It is also possible that the uptake of cancer screening is improving in women with IDD, as smaller disparities are reported in the most recent studies. However, the number of population- based studies is insufficient to draw conclusions.

Differences observed between women with and without IDD remained when controlling for potential confounders (age, socio-economic status, rurality, and expected use of healthcare resources).

Consequently, findings suggest that women with IDD face important health inequities in missing opportunities for the prevention and early detection of cervical and breast cancer. Since the introduction of cervical and breast cancer screening to routine preventative care, media campaigns have increased public awareness about their relevance. Observed inequities reveal an urgent need for more intensive and group-specific or individually tailored strategies that can remove barriers to screening (Finney Rutten et al. 2004). Barriers to cancer screening for women with IDD are multiple, and targeted strategies should thus be multifaceted (Willis et al. 2008). Further research is needed to unpack the challenges faced by women with IDD when accessing cervical and breast cancer screening, and to provide recommendations on how to decrease inequities.

Logistic and practical barriers have been reported to limit the accessibility to cancer screening in women with IDD. For example, transportation to healthcare facilities may be challenging for some women (Willis et al. 2008). The equipment used in both cervical and breast cancer screening is not adapted to those with a physical disability (Willis et al. 2008; Tyler et al. 2010). Women with IDD might have problems standing during the mammogram or transferring from a wheelchair to the consultation table. From the perspective of women with IDD, technicians and health professionals should spend more time with them to accommodate their needs and provide information on the procedure (Wilkinson et al. 2011). Training on available adaptations and supports to the procedures (e.g. safe transfer to the exam table, alternative positioning for the pelvic exam) appear to be needed.

Previous research has highlighted the lack of knowledge and training of healthcare professionals and technicians on the health and support needs of women with IDD (Willis et al. 2008). One important issue is the belief that women with IDD are not at risk of cervical or breast cancer, and thus screening is not required. However, women with IDD now live until the age of 71 years old on average, and thus are at risk for age-related diseases (Bittles et al. 2002). In addition, Pap smears are only recommended for women who have been sexually active (Cancer Care Ontario 2000), but women with IDD might find it difficult to communicate their sexual history and may have experienced non- consensual sexual activity that they cannot or will not admit to (Reynolds et al. 2008). Neither this study nor previously published 
studies showing differences in cervical cancer uptake for women with IDD have been able to control for this factor.

However, it is documented that many women with IDD experience sexual relationships (Gesualdi 2006; Wood \& Douglas 2007; Reynolds et al. 2008), and thus differences observed are unlikely to reflect a lower proportion of women who have been sexually active among the IDD cohort. In the case of breast cancer, a lower incidence of cancer has been reported in women with Down syndrome (Willis et al. 2008). The overall rate of screening in the IDD group would understandably be lower than in the general population if providers do not refer women with Down syndrome for screening. It would be important to account for this factor in future studies. It would also be relevant to examine if healthcare providers make the assumption that all women with IDD are protected from breast cancer, not only those with Down syndrome.

Another important barrier to cancer screening is the challenge of seeking valid consent from women with IDD (Kirby \& Hegarty 2010), when seeking consent is mandatory for any medical procedure, and especially one of an invasive nature such as the Pap test. Women with IDD report a lack of knowledge about cervical and breast cancer screening, and their fears and embarrassment impede the procedure (Truesdale-Kennedy et al. 2011; Wilkinson et al. 2011; Parish et al. 2012b). Interventions tailored to the communication skills of women with IDD are required in order to increase their knowledge of the procedure and its benefits, decrease their anxiety, and thus allow them to give informed consent (e.g. Broughton 2002; Lunsky et al. 2003; Biswas et al. 2005; Parish et al. 2012a). The role of family caregivers and paid staff is crucial in providing information on cervical and breast cancer screening, supporting the person during the procedure and reporting any potential symptoms of cancer to health professionals (Hanna et al. 2011; Taggart et al. 2011). Information and training are required to support caregivers and staff in this role.

Cancer screening initiatives need to specifically consider vulnerable populations such as women with IDD when planning their strategies. One-to- one counselling with women with IDD is an intervention that has shown promise (Biswas et al. 2005) and requires further study. A recent randomised control trial demonstrated the positive effect of a curriculum (Women be Healthy; Lunsky et al. 2003) aimed at increasing knowledge on cervical and breast cancer screening in women with IDD (Parish et al. 2012a). However, the impact of such programmes at the population level has yet to be studied. Training and information must be provided to healthcare professionals on the importance of health screening in persons with IDD and on how to support their patients in understanding and consenting to the procedure (McIlfatrick et al. 2011; Parish et al. 2012a). Developing clinical guidelines and tools, such as the Canadian Consensus Guide- lines on the primary care of adults with IDD (Sullivan et al. 2011), might be useful in this regard.

Study strengths and limitations

This is the first population based study controlling for age, rurality, income quintile and expected use of healthcare resources to examine cancer screening rates in women with IDD compared with the general population. Other factors might impact on the receipt of cancer screening but could not be addressed using the specified sources of data. For example, living arrangements or levels of IDD are not included in the health administrative datasets consulted. However, these factors are more relevant when comparing 
different IDD groups with each other than when comparing an IDD group with the general population. The magnitude of the differences we found suggest that even if we had been able to measure residential status or IDD severity, this would not erase the very large gap that we found between individuals with IDD and our general population sample in terms of their access to cancer screening. Further study may examine factors influencing the receipt of cancer screening among groups with IDD. Immigration status and racial identity are other variables which may impact cancer screening utilisation and were not available to be included in this study.

Relying on large administrative datasets presents many advantages including increased representation and lack of recall bias. However, it is likely that not all eligible women with IDD were identified using the methods described. The strengths and limitations of the algorithm used to identify persons with IDD in the health administrative datasets is dis- cussed elsewhere (Lin et al. 2012). Through our case identification method, the prevalence rate of IDD in the adult population living in Ontario has been established at 0.52 , which is likely to be an underestimation. Our case finding approach possi- bly identifies fewer people with mild forms of IDD as physicians might be less likely to identify this condition when filing a billing claim.

Conclusion

This paper supports previous evidence that women with IDD are facing inequities when accessing cervical and breast cancer screening. It is time to provide evidence of the effectiveness of interventions promoting preventative care in persons with IDD. 


\section{Conflict of interest}

No conflicts of interest have been declared.

Acknowledgements

This study was funded by the Canadian Institutes of Health Research Partnerships in Health Systems Improvement grant (PHE\# 103973) in partnership with the Ontario Ministry of Community and Social Services and Surrey Place Centre, and is part of the Health Care Access Research in Developmental Disabilities (H-CARDD) Program. The work was also supported by the Centre for Addiction and Mental Health (CAMH) and the Institute for Clinical Evaluative Sciences (ICES), which receive annual grants from the Ontario Ministry of Health and Long Term Care (MOHLTC), as well as by CAMH and Fonds de Recherche en Sante du Quebec post-doctoral support for R. Balogh and V. Cobigo. The opinions, results and conclusions reported in this paper are those of the authors and are not necessarily those of the funding sources. No endorsement by CIHR, CAMH, ICES, the Ontario MOHLTC or the Fonds de Recherche en Sante du Quebec is intended or should be inferred. The authors gratefully acknowledge the assistance and support of Jennifer Bennie and Drew Wilton at the Institute for Clinical Evaluative Sciences. 


\section{References}

Biswas M., Whalley H., Foster J., Friedman E. \& Deacon R. (2005) Women with learning disability and uptake of screening: audit of screening uptake before and after one to one counselling. Journal of Public Health 27, 344-7.

Bittles A. H., Petterson B. A., Sullivan S. G., Hussain R., Glasson E. J. \& Montgomery P. D. (2002) The influence of intellectual disability on life expectancy. The Journals of Gerontology. Series A, Biological Sciences and Medical Sciences 57, M470-M472.

Boyle P. \& Levin B. (eds) (2008) World Cancer Report 2008. International Agency for Research on Cancer, Lyon.

Broughton S. (2002) A review of the literature: interventions to maximize capacity to consent and reduce anxiety of women with learning disabilities preparing for a cervical smear test. Health Services Management Research 15, 173-85.

Cancer Care Ontario (2000) Ontario Cervical Screening Program: Program Report 1997-2000. Cancer Care Ontario, Toronto.

Cancer Care Ontario (2010) Ontario Breast Screening Program: 20th Anniversary Report 1990-2010. Cancer Care Ontario, Toronto.

CHSPR (2008) Measuring morbidity in populations: per- formance of the Johns Hopkins Adjusted Clinical Group Case-Mix Adjustment System in Manitoba, 1-90.

Davies N. \& Duff M. (2001) Breast cancer screening for older women with intellectual disability living in com- munity group homes. Journal of Intellectual Disability Research 45, 253-7.

Eaker S., Adami H. O. \& Sparen P. (2001) Reasons women do not attend screening for cervical cancer: a population-based study in Sweden. Preventive Medicine 32, 482-91.

Finney Rutten L. J., Nelson D. E. \& Meissner H. I. (2004) Examination of population-wide trends in barriers to cancer screening from a diffusion of innovation perspective (1987-2000). Preventive Medicine 38, 258-68.

Gesualdi G. (2006) Screening for cancer in women with developmental disabilities: are they receiving the appropriate standard of care? The Exceptional Parent 36, 61-5.

Hanna L. M., Taggart L. \& Cousins W. (2011) Cancer prevention and health promotion for people with intellectual disabilities: an exploratory study of staff knowledge. Journal of Intellectual Disability Research 55, 281-91.

Havercamp S. M., Scandlin D. \& Roth M. (2004) Health disparities among adults with developmental disabilities, adults with other disabilities, and adults not reporting disability in North Carolina. Public Health Reports (Washington, D.C.: 1974) 119, 418-26. 
Kirby S. \& Hegarty J. (2010) Breast awareness within an intellectual disability setting. European Journal of Oncology Nursing 14, 328-36.

Lalor A. \& Redmond R. (2009) Breast screening for post- menopausal women. Learning Disability Practice 12, 28-33.

Lin E., Balogh R., Cobigo V., Ouellette-Kuntz H., Wilton A.S. \& Lunsky Y. (2012) Using administrative health data to identify individuals with intellectual and developmental disabilities: a comparison of algorithms. Journal of Intellectual Disability Research. doi: 10.1111/ jir.12002

Lofters A., Glazier R. H., Agha M. M., Creatore M. \& Moineddin R. (2007) Inadequacy of cervical cancer screening among urban recent immigrants: a population-based study of physician and laboratory claims in Toronto, Canada. Preventive Medicine 44, 536- 42.

Lofters A. K., Moineddin R., Hwang S. W. \& Glazier R. H. (2010) Low rates of cervical cancer screening among urban immigrants: a population-based study in Ontario, Canada. Medical Care 48, 611-18.

Lunsky Y., Stariko A. \& Armstrong S. C. (2003) Women Be Healthy: evaluation of a women's health curriculum for women with intellectual disabilities. Journal of Applied Research in Intellectual Disabilities 16, 247-53.

McIlfatrick S., Taggart L. \& Truesdale-Kennedy M. (2011) Supporting women with intellectual disabilities to access breast cancer screening: a healthcare professional perspective. European Journal of Cancer Care 20, 412-20.

Parish S. L. \& Saville A. W. (2006) Women with cognitive limitations living in the community: evidence of disability-based disparities in health care. Mental Retar- dation 44, 249-59.

Parish S. L., Rose R. A., Luken K., Swaine J. G. \& O’Hare L. (2012a) Cancer screening knowledge changes: results from a randomized control trial of women with develop- mental disabilities. Research on Social Work Practice 22, 43-53.

Parish S. L., Swaine J. G., Luken K., Rose R. A. \& Dabab- nah S. (2012b) Cervical and breast cancer screening knowledge of women with developmental disabilities. Intellectual and Developmental Disabilities 50, 79-91.

Reynolds F., Stanistreet D. \& Elton P. (2008) Women with learning disabilities and access to cervical screening: retrospective cohort study using case control methods. BMC Public Health 8, 30.

Service Ontario (2008) Services and Supports to Promote the Social Inclusion of Persons with Developmental Dis- abilities Act, 2008, c. 14.

Spadea T., Bellini S., Kunst A., Stirbu I. \& Costa G. (2010) The impact of interventions to improve attend- ance in female cancer screening among lower socioeconomic groups: a review. Preventive Medicine 50, 159-64. 
Statistics Canada (2007) Standard Geographical Classification (SGC):Volume 1 - The Classification. Catalogue no. 12-571-XIE. Ministry of Industry, Ottawa.

Statistics Canada (2009) Dissemination area (DA). Avail- able at: http://www12.statcan.ca/censusrecensement/ 2006/ref/dict/geo021-eng.cfm (retrieved 22 July 2011).

Stein K. \& Allen N. (1999) Cross-sectional survey of cer- vical cancer screening in women with learning disability. British Medical Journal 318, 641.

Sullivan S. G., Glasson E. J., Hussain R., Petterson B. A., Slack-Smith L. M., Montgomery P. D. et al. (2003) Breast cancer and the uptake of mammography screen- ing services by women with intellectual disabilities. Pre- ventive Medicine 37, 507-12.

Sullivan W. F., Berg J. M., Bradley E., Cheetham T., Denton R., Heng J. et al. (2011) Primary care of adults with developmental disabilities: Canadian consensus guidelines. Canadian Family Physician 57, 541-53.

Taggart L., Truesdale-Kennedy M. \& McIlfatrick S. (2011) The role of community nurses and residential staff in supporting women with intellectual disability to access breast screening services. Journal of Intellectual Disability Research 55, 41-52.

The Johns Hopkins University Bloomberg School of Public Health (2005) The Johns Hopkins ACG Case- Mix System: Reference Manual Version 7.0, 1-214.

Truesdale-Kennedy M., Taggart L. \& McIlfatrick S. (2011) Breast cancer knowledge among women with intellectual disabilities and their experiences of receiving breast mammography. Journal of Advance Nursing 67, 1294-304.

Tyler C. V., Zyzanski S. J., Panaite V. \& Council L. (2010) Nursing perspective on cancer screening in adults with intellectual and other developmental disabilities. Intellec- tual and Developmental Disabilities 48, 271-7.

Wilkinson J. E., Deis C. E., Bowen D. J. \& Bokhour B. G. (2011) 'It’s easier said than done’: perspective on mam- mography from women with intellectual disabilities. Annals of Family Medicine 9, 142-7.

Willis D. S., Kennedy C. M. \& Kilbride L. (2008) Breast cancer screening in women with learning disabilities: current knowledge and considerations. British Journal of Learning Disabilities 36, 171-84.

Wood R. \& Douglas M. (2007) Cervical screening for women with learning disability: current practice and attitudes within primary care in Edinburgh. British Journal of Learning Disabilities 35, 84-92. 